\title{
ESTIMATES FOR FUNDAMENTAL SOLUTIONS OF SECOND-ORDER SUBELLIPTIC DIFFERENTIAL OPERATORS
}

\author{
MICHAEL CHRIST
}

(Communicated by Barbara L. Keyfitz)

\begin{abstract}
A simple proof is given of pointwise estimates of C. Fefferman and A. Sánchez-Calle for the fundamental solution of a subelliptic, second-order partial differential operator with nonnegative characteristic form, based on a rescaling argument.
\end{abstract}

\section{INTRODUCTION}

Let $M$ be a $C^{\infty}$ smooth, compact manifold of dimension $n$ and $L$ be a second-order differential operator on $M$ with real, $C^{\infty}$ coefficients. $L$ is said to have nonnegative characteristic form if its principal symbol is a nonpositive function on the cotangent bundle of $M$. It is said to be subelliptic if there exists $\varepsilon>0$ such that whenever $\eta, \psi \in C^{\infty}(M)$ and $\eta \psi \equiv \eta$, then for all $0 \leq s \in \mathbb{R}$ and all $f \in C^{\infty}(M)$

$$
\|\eta f\|_{L_{s+k}^{2}} \leq C(\eta, \psi)\|\psi L f\|_{L_{s}^{2}}+C(\eta, \psi)\|\psi f\|_{2}
$$

where $L_{s}^{2}$ denotes the Sobolev space of all functions possessing $s$ derivatives in $L^{2}$ and $\|f\|_{p}$ denotes the $L^{p}$ norm, with respect to some fixed positive measure with a smooth, nowhere vanishing density on $M$.

We assume henceforth that $L$ is subelliptic with nonnegative characteristic form. The purpose of this note is to present a second proof of estimates due to Fefferman and Sánchez-Calle [FS] for the fundamental solutions of such operators. We believe that the new proof may be a bit simpler, both technically and conceptually.

Fefferman and Sánchez-Calle associate to $L$ a metric $\rho(x, y)$ (in the sense of point-set topology) on $M$, a family of open "balls" $B(x, r)$ for $(x, r) \in$ $M \times\left(0, r_{0}\right]$ (called standard balls in [FS]) satisfying

$$
\left\{y: \rho(x, y)<C^{-1} r\right\} \subset B(x, r) \subset\{y: \rho(x, y)<C r\},
$$

Received by the editors January 8, 1988.

1980 Mathematics Subject Classification (1985 Revision). Primary 35A08, 35H05.

Research supported by an NSF grant and the Mathematical Sciences Research Institute. 
and for each $(x, r)$ a diffeomorphism $\Phi_{x, r}: Q \leftrightarrow B(x, r)$ where $Q$ denotes the unit cube in $\mathbb{R}^{n}$. It follows from the hypotheses that the formal adjoint $L^{*}$ is also subelliptic. Therefore there exists $K: \mathscr{D}^{\prime}(M) \mapsto \mathscr{D}^{\prime}(M)$ such that

$$
L K=I+S \quad \text { and } \quad K L=I+S^{\prime}
$$

on $L^{2}$, where $I$ is the identity and $S, S^{\prime}$ map $\mathscr{D}^{\prime}$ boundedly to $\mathscr{D}=C^{\infty}$. The distribution-kernel $k(x, y)$ of $K$ is $C^{\infty}$ off of the diagonal. [FS] quantified this:

Theorem. Assume $n \geq 3$. Then

$$
|k(x, y)| \leq C \rho(x, y)^{2}|B(x, \rho(x, y))|^{-1}
$$

for all $x \neq y \in M$. More precisely, for each $\varepsilon>0$ and $N$ there exists $C$ such that for all $x \in M$ and $r \in\left(0, r_{0}\right]$,

$$
r^{-2}|B(x, r)| \cdot\|k(\Phi x, \Phi y)\|_{C^{N}\{(x, y) \in Q \times Q:|x-y|>\varepsilon\}} \leq C .
$$

$\Phi$ denotes $\Phi_{x, r}$. Illustrative examples may be found in [FS]; see also [NSW]. In the selfadjoint case the right-hand member in (a) is also a lower bound for $k$, up to a constant factor [FS].

We shall derive this result as a consequence of the known fundamental properties of $L, \rho,\left\{B(x, r), \Phi_{x, r}\right\}$ together with simple and rather formal arguments. In $\S 2$ we review the relevant properties, and in $\S 3$ present the proof. Three disclaimers: First, our proof is not so much shorter as it might appear, since we quote extensively from [FS] in $\S 2$. Second, the more elaborate machinery of [FS] appears also to be more powerful, so may well prove to be more useful for other purposes. Third, our approach has the same basis as [FS], the exploitation of uniformity under rescaling.

One of our motivations was the ingenious application of these estimates to the study of the Kohn Laplacian on certain CR manifolds made by Machedon $[\mathrm{M}]$.

Thanks are due to $\mathrm{S}$. Chanillo for helpful conversations.

\section{REVIEW}

In local coordinates write

$$
L=\sum_{i, j} a_{i, j} \partial_{i, j}^{2}+\sum b_{l} \partial_{l}+c
$$

with $\left(a_{i, j}\right)$ symmetric and nonnegative. $L$ is subelliptic if and only if the system of vector fields $\sum b_{l} \partial_{l}, \sum a_{1, j} \partial_{j}, \ldots, \sum a_{n, j} \partial_{j}$ satisfies the condition of Hörmander, that the Lie algebra they generate should span the tangent space at each point [FS, OR]. When $L$ is selfadjoint with respect to the given measure on $M, \rho$ is defined as follows [FP]: $\xi=\sum \xi_{j} \partial_{j}$ is said to be subunit at $x$ if $\left(\xi_{i} \xi_{j}\right) \leq\left(a_{i, j}(x)\right)$ as quadratic forms. Then $\rho(x, y) \leq r$ if and only if there exists a Lipschitz path $\gamma:[0, r] \mapsto M$ with $\gamma(0)=x, \gamma(r)=y$, and $\gamma^{\prime}(t)$ 
subunit at $\gamma(t)$ for almost all $t$. For the extension to general $L$, and for the definition of the standard balls $B(x, r)$ and rescaling maps $\Phi_{x, r}$, see [FS].

Assuming always that $L$ is subelliptic with nonnegative characteristic form we list basic facts, for which proofs may for the most part be found in [FS and FP].

$$
C^{-1}|x-y| \leq \rho(x, y) \leq C|x-y|^{\varepsilon} \quad \text { for all } x, y \in M
$$

for some $C<\infty, \varepsilon>0$.

$\mid$ determinant $\left(\partial \Phi_{x, r}\right)(y)|\sim| B(x, r) \mid$ uniformly in $(x, r, y) \in M \times\left(0, r_{0}\right] \times Q$, and for all $y \in Q$ and multi-indices $\alpha$

$$
\mid \partial_{y}^{\alpha} \text { determinant }\left(\partial \Phi_{x, r}\right)(y)\left|\leq C_{\alpha}\right| B(x, r) \mid
$$

while for all $\delta>0$

$$
\left|\partial_{y}^{\alpha}\left(\Phi_{x, r}^{-1} \circ \Phi_{z, \delta r}\right)(y)\right| \leq C(\alpha, \delta)
$$

on the domain of the composition.

Since $n \geq 3$ there exists $\delta>0$ (in fact $\delta \geq 1$ ) such that

$$
\begin{gathered}
|B(x, t r)| \leq C t^{2+\delta}|B(x, r)| \text { for all } t \leq 1 . \\
|B(x, 2 r)| \sim|B(x, r)|
\end{gathered}
$$

and

$$
\left|B\left(x, r_{0}\right)\right| \sim 1 \text {. }
$$

In all such estimates we assert tacitly that the constants involved are uniform in $x, r$.

Let $(x, r) \in M \times\left(0, r_{0}\right]$, set $\Phi=\Phi_{x, r}$ and for $f \in C_{0}^{2}(Q)$ define

$$
L_{\Phi} f=r^{2} L\left(f \circ \Phi^{-1}\right) \circ \Phi \text {. }
$$

The fundamental property of the maps $\Phi$ is that $L_{\Phi}$ is subelliptic, uniformly in $x, r$. Thus there exist $\widehat{\mathscr{P}}_{\Phi}, \widehat{\mathscr{E}}_{\Phi}$ defined on those $f \in L^{2}(Q)$ supported in $Q / 4$, mapping to $L^{2}$ functions supported in $Q / 2$, such that (uniformly in $x, r)$

$$
\begin{aligned}
& L_{\Phi} \widehat{\mathscr{P}}_{\Phi}=I-\widehat{\mathscr{E}}_{\Phi}, \\
& \widehat{\mathscr{P}}_{\Phi}, \widehat{\mathscr{E}}_{\Phi} \text { are bounded in } L^{2} \text { operator norm, } \\
& \widehat{\mathscr{E}}_{\Phi} \text { is smoothing of infinite order }
\end{aligned}
$$

and for any $\eta, \psi \in C_{0}^{\infty}(Q)$ with $\eta \psi \equiv \eta$, for any $s \geq 0$,

$$
\|\eta f\|_{L_{s}^{2}} \leq C\left\|\psi L_{\Phi} f\right\|_{L_{s}^{2}}+C\|\psi f\|_{2}
$$

with $C$ dependent only on $L, s$ and on upper bounds on derivatives of $\eta, \psi$. (2.7) is not explicit in [FS] but follows directly from their Corollary, page 254. 
Transfer $\widehat{\mathscr{P}}_{\Phi}$ and $\hat{\mathscr{E}}_{\Phi}$ to operators on $M$ by defining for each standard ball $B=B(x, r)$, for each $f \in L^{2}(B)$ supported in $\Phi(Q / 4)$ (where $\Phi=\Phi_{x, r}$ )

$$
\mathscr{P}_{B} f=r^{2} \widehat{\mathscr{P}}_{\Phi}(f \circ \Phi) \circ \Phi^{-1}
$$

and

$$
\mathscr{E}_{B} f=\hat{\mathscr{E}}_{\Phi}(f \circ \Phi) \circ \Phi^{-1}
$$

so that $L \mathscr{P}_{B}=I-\mathscr{C}_{B}$. Then

$$
\begin{aligned}
\left\|\mathscr{P}_{B} f\right\|_{2} & \leq C r^{2}\|f\|_{2}, \\
\left\|\mathscr{E}_{B} f\right\|_{\infty} & \leq C_{0}|B|^{-1}\|f\|_{1}
\end{aligned}
$$

and there exists $N$, depending only on $L$, so that

$$
\left\|\mathscr{P}_{B} f\right\|_{\infty} \leq C r^{2}\|f\|_{C^{N}(B)}
$$

for all $f \in C^{N}(M)$ supported in $\Phi(Q / 4)$. Here $\|g\|_{C^{N}(B)}$ is defined to be $\|g \circ \Phi\|_{C^{N}(Q)}$. Finally $K$ itself is bounded on $L^{2}(M)$.

\section{Proof}

As in the proof in [C] of analogous estimates for the Szegö projection, the main point is a weak fractional integration inequality.

Proposition. There exists $C<\infty$ such that for all $x \in M$ and $r \in\left(0, r_{0}\right]$, for all $f \in L^{2}$ supported in $B(x, r)$,

$$
\|K f\|_{L^{2}(B(x, r))} \leq C r^{2}\|f\|_{2}
$$

Proof. To simplify notation we set $r_{0}=1$. Fix $\theta$, a small positive number to be chosen below. Let $r$ be given. Specify $m \in \mathbb{Z}^{+}$by $\theta^{m+2} \leq r<\theta^{m+1}$. For $0 \leq j \leq m+1$ let $\mathscr{P}_{j}=\mathscr{P}_{B_{j}}, B_{j}=B\left(x, \theta^{j}\right), \mathscr{E}_{j}=\mathscr{E}_{B_{j}}$. Then for any $f \in L^{2}(B(x, r))$,

$$
K f=K L \mathscr{P}_{m} f+K \mathscr{E}_{m} f=(I+S) \mathscr{P}_{m} f+K \mathscr{E}_{m} f .
$$

$\mathscr{E}_{m} f$ is supported in $B_{m}$, which is well inside $B_{m-1}$ if $\theta$ is small enough, so that

$$
\mathscr{E}_{m} f=L \mathscr{P}_{m-1} \mathscr{E}_{m} f+\mathscr{E}_{m-1} \mathscr{E}_{m} f .
$$

Substituting into (3.1) and iterating yields eventually

$$
K f=(I+S)\left[\mathscr{P}_{m}+\mathscr{P}_{m-1} \mathscr{E}_{m}+\cdots+\mathscr{P}_{0} \mathscr{E}_{1} \cdots \mathscr{E}_{m}\right] f+K \mathscr{E}_{0} \mathscr{E}_{1} \ldots \mathscr{E}_{m} f
$$

The main issue is the behavior of $\mathscr{E}_{i} \cdots \mathscr{E}_{m} f$ for $0 \leq i \leq m$. Since $\mathscr{E}_{j+1} \cdots \mathscr{E}_{m} f$ is supported in $B_{j+1}$,

$$
\begin{aligned}
\left\|\mathscr{E}_{j} \ldots \mathscr{E}_{m} f\right\|_{\infty} & \leq C_{0}\left|B_{j}\right|^{-1}\left\|\mathscr{E}_{j+1} \cdots \mathscr{E}_{m} f\right\|_{1} \\
& \leq C_{0}\left(\left|\boldsymbol{B}_{j+1} /\right| \boldsymbol{B}_{j} \mid\right)\left\|\mathscr{E}_{j+1} \cdots \mathscr{E}_{m} f\right\|_{\infty}
\end{aligned}
$$


so that by induction

$$
\left\|\mathscr{E}_{j} \ldots \mathscr{E}_{m} f\right\|_{\infty} \leq C_{0}^{m-j+1}\left|B_{j}\right|^{-1}\|f\|_{1}
$$

and by one more iteration

$$
\left\|\mathscr{E}_{i} \cdots \mathscr{E}_{m} f\right\|_{C^{N}\left(B_{i}\right)} \leq C_{N} C_{0}^{m-i+1}\left|B_{i}\right|^{-1}\|f\|_{1} .
$$

If $N$ is chosen large enough then (2.11) gives

$$
\left\|\mathscr{P}_{i} \mathscr{E}_{i+1} \cdots \mathscr{E}_{m} f\right\|_{\infty} \leq C C_{0}^{m-i} \theta^{2 i}\left|B_{i}\right|^{-1}\|f\|_{1}
$$

whence by Hölder's inequality (twice)

$$
\begin{aligned}
\left\|\mathscr{P}_{i-1} \mathscr{E}_{i} \cdots \mathscr{E}_{m} f\right\|_{L^{2}\left(B_{m+1}\right)} & \leq C C_{0}^{m-i} \theta^{2 i}\left(\left|B_{m+1}\right| /\left|B_{i}\right|\right)\|f\|_{2} \\
& \leq C C_{0}^{m-i} \theta^{2 i} \theta^{(m+1-i)(2+\delta)}\|f\|_{2} \\
& \leq C_{\theta}\left(C_{0} \theta^{\delta}\right)^{m-i} \theta^{2 m}\|f\|_{2} .
\end{aligned}
$$

Since $\delta>0$ we may choose $\theta$ so that $C_{0} \theta^{\delta}<1$. Since $\left\|\mathscr{P}_{m} f\right\|_{2} \leq C \theta^{2 m}\|f\|_{2}$ and $\theta^{2 m} \sim r^{2}$, the triangle inequality yields

$$
\left\|\left[\mathscr{P}_{m}+\mathscr{P}_{m-1} \mathscr{E}_{m}+\cdots+\mathscr{P}_{0} \mathscr{E}_{1} \ldots \mathscr{E}_{m}\right] f\right\|_{L^{2}\left(B_{m+1}\right)} \leq C r^{2}\|f\|_{2} .
$$

Moreover since $S$ is smoothing of infinite order,

$$
\begin{aligned}
& \left\|S\left[\mathscr{P}_{m}+\cdots+\mathscr{P}_{0} \mathscr{E}_{1} \ldots \mathscr{E}_{m}\right] f\right\|_{L^{2}\left(B_{m+1}\right)} \\
& \leq C\left|B_{m+1}\right|^{1 / 2}\left(\left\|\mathscr{P}_{m} f\right\|_{1}+\sum_{i=0}^{m-1}\left\|\mathscr{P}_{i} \mathscr{E}_{i+1} \ldots \mathscr{E}_{m} f\right\|_{1}\right) \\
& \leq C\left|B_{m+1}\right| r^{2}\|f\|_{2}+C \sum_{i=1}^{m-1} C_{0}^{m-i} \theta^{2 i}\left|B_{m+1}\right|\|f\|_{2} \\
& \leq C r^{2}\|f\|_{2}
\end{aligned}
$$

by comparison with the derivation of (3.4). Finally

$$
\begin{aligned}
\left\|K \mathscr{E}_{0} \ldots \mathscr{E}_{m} f\right\|_{L^{2}\left(B_{m+1}\right)} & \leq\left|B_{m+1}\right|^{1 / 2}\left\|K \mathscr{E}_{0} \ldots \mathscr{E}_{m} f\right\|_{L^{\infty}(M)} \\
& \leq C\left|B_{m+1}\right|^{1 / 2}\left\|\mathscr{E}_{0} \ldots \mathscr{E}_{m} f\right\|_{C^{N}(M)} \\
& \leq C\left|B_{m+1}\right| C_{0}^{m}\|f\|_{2} \\
& \leq C \theta^{2 m}\|f\|_{2}
\end{aligned}
$$

since $C_{0} \theta^{\delta}<1$. We have used (3.3) with $i=0$ and the fact that $|B(y, 1)| \sim 1$ uniformly for $y \in M$.

To deduce the Theorem from the Proposition let $z \in M, r \leq 1$ be arbitrary and let $\Phi: Q \mapsto B(z, r)$ be the rescaling map. For compactly supported $f \in$ $L^{2}(Q)$ set

$$
\begin{aligned}
K_{\Phi} f & =r^{-2} K\left(f \circ \Phi^{-1}\right) \circ \Phi \\
S_{\Phi}^{\prime} f & =S^{\prime}\left(f \circ \Phi^{-1}\right) \circ \Phi
\end{aligned}
$$


where $f \circ \Phi^{-1}$ is viewed as an element of $L^{2}(M)$, identically zero outside $B(z, r)$, so that

$$
K_{\Phi} L_{\Phi}=I+S_{\Phi}^{\prime}
$$

Since $|\operatorname{det}(D \Phi)| \sim|B(z, r)|$ on $Q$, it follows from the Proposition that

$$
\left\|K_{\Phi} f\right\|_{2} \leq C\|f\|_{2} \text { for all } f \in L^{2}(Q)
$$

uniformly in $z, r$. Furthermore by (2.2) $S_{\Phi}^{\prime}$ is given by integration against a $C^{\infty}$ kernel whose $C^{N}$ norm is $O\left(C_{N}|B(z, r)|\right) \leq C_{N}^{\prime}$ for all $N, z, r$.

Let $\varepsilon>0$ be small and consider any two Euclidean balls $Q_{1}, Q_{2}$ of radii $\varepsilon$, separated from $\partial Q$ and from one another by a distance of at least $\varepsilon$. Choose $\eta_{0}, \eta_{1} \in C_{0}^{\infty}(Q)$, supported in $\{$ distance $(x, \partial Q) \geq \varepsilon / 2\}$, with $\eta_{i} \equiv 1$ on $Q_{2}$, $\eta_{i} \equiv 0$ on $Q_{1}$ for both $i$, and $\eta_{0} \eta_{1} \equiv \eta_{0}$ and $\left\|\eta_{i}\right\|_{C^{N}} \leq C(N, \varepsilon)$ for all $N$. Then for any $f \in L^{2}\left(Q_{1}\right)$ and any $s \geq 0$, by (2.8),

$$
\begin{aligned}
\left\|\eta_{0} K_{\Phi} f\right\|_{L_{s}^{2}} & \leq C_{s, \varepsilon}\left\|\eta_{1} L_{\Phi} K_{\Phi} f\right\|_{L_{s}^{2}}+C_{s, \varepsilon}\left\|\eta_{1} K_{\Phi} f\right\|_{2} \\
& \leq C_{s, \varepsilon}\left\|\eta_{1}\left(I+S_{\Phi}\right) f\right\|_{L_{s}^{2}}+C_{s, \varepsilon}\|f\|_{2} \\
& \leq C_{s, \varepsilon}\|f\|_{2} .
\end{aligned}
$$

Therefore if $k_{\Phi}$ is the distribution-kernel for $K_{\Phi}$, so that

$$
K_{\Phi} f(x)=\int k_{\Phi}(x, y) f(y) d y
$$

in the sense of distributions, the Sobolev embedding lemma gives

$$
\left\|\partial_{x}^{\alpha} k_{\Phi}(x, \cdot)\right\|_{L^{2}\left(Q_{1}\right)} \leq C_{\alpha, \varepsilon} \quad \text { uniformly in } x \in Q_{2} \text {. }
$$

Now $L^{*}$ is not only subelliptic, but has associated to it the same family of balls and rescaling maps as $L$. Therefore by virtue of (2.1) and (2.2), the same argument applies to the kernel $\bar{k}_{\Phi}(y, x)$ :

$$
\left\|\partial_{y}^{\alpha} k_{\Phi}(\cdot, y)\right\|_{L^{2}\left(Q_{2}\right)} \leq C_{\alpha, \varepsilon} \quad \text { uniformly in } y \in Q_{1} \text {. }
$$

It results from (3.5), (3.6) and the Sobolev embedding lemma that $k_{\Phi} \in$ $C^{\infty}\left(Q_{2} \times Q_{1}\right)$, uniformly in $z, r$. By (2.1) and (2.2) this is the conclusion of the Theorem.

\section{REFERENCES}

[C] M. Christ, Regularity properties of the $\bar{\partial}_{b}$ equation on weakly pseudoconvex $C R$ manifolds of dimension 3, J. Amer. Math. Soc. 1 (1988), 587-646.

[FP] C. L. Fefferman and D. H. Phong, Subelliptic eigenvalue problems, Proceedings of Conference on Harmonic Analysis in Honour of Antoni Zygmund (1981), 590-606, Wadsworth Mathematics Series.

[FS] C. L. Fefferman and A. Sánchez-Calle, Fundamental solutions for second order subelliptic operators, Ann. of Math. 124 (1986), 247-272.

[M] M. Machedon, Estimates of the parametrix of the Kohn Laplacian on certain weakly pseudoconvex domains, preprint. 
[NSW] A. Nagel, E. M. Stein and S. Wainger, Balls and metrics defined by vector fields I: Basic properties, Acta Math. 155 (1985), 103-147.

[OR] O. Oleinik and E. Radkevitch, "Second-order equations with nonnegative characteristic form," Amer. Math. Soc., Providence, R.I., 1973.

Department of Mathematics, University of California, Los ANgeles, California 90024 